\title{
A PROPER-MOTION SEARCH FOR DWARF CARBON AND S STARS
}

D. J. MacCONNELL

Computer Sci. Corp./Space Telescope Science Institite

Baltimore, MD 21218, U.S.A.

R. L. WILLIAMSON II

Space Telescope Science Institute

Baltimore, MD 21218, U.S.A.

AND

W. J. ROBERTS

Box 16329, Baltimore, MD 21210, U.S.A.

\begin{abstract}
We report on an effort to identify further members of the dwarf carbon class and new members of a putative dwarf $S$ group in the general field through determination of proper motions of catalogued stars. Examination of nearly $1500 \mathrm{C}$ stars and over $300 \mathrm{~S}$ stars reveals some interesting false alarms but no new dwarf members of these classes.
\end{abstract}

\section{Introduction}

The majority of galactic carbon stars have been found on objective-prism photographic plates of low resolution/dispersion taken with wide-angle cameras in any of several spectral bands from the blue to the near-infrared. In the blue-visual region, the Swan bands of $\mathrm{C}_{2}$ are the defining criteria for membership in the class, while in the red and near-IR, the great strength of the $\mathrm{CN}$ bands is taken as sufficient for inclusion in the class. Having such evolved atmospheres, all $\mathrm{C}$ stars are expected to be on the AGB, and their high luminosities and distinctive optical spectra make them excellent probes of the kinematics and structure of the Galaxy to large distances. A challenge to this scenario, however, came in 1977 with the realization that the high-proper-motion star G77-61 was a normal-appearing C star but of $M_{V} \sim+10$ (Dahn et al. 1977), and it became the prototype of the dwarf carbon $(\mathrm{dC})$ class. Since stars of dwarf mass cannot produce 
and dredge up carbon to the photosphere, G77-61 was explained as the product of mass transfer in a close binary of large mass ratio - the $\mathrm{dC}$ has received its carbon from an originally more massive AGB companion which is now a white dwarf. Indeed, Dearborn et al. (1986) found that G77-61 is a single-lined spectroscopic binary of $P=245$ days and that the undetected, degenerate companion must have $T<6000 \mathrm{~K}$. It remained the only $\mathrm{dC}$ until several others were identified early in this decade via their proper motions (Green et al. 1991, 1992). To date, 11 stars have been presented as dCs, and two of these, PG 0824+289 (Heber et al. 1993) and CBS 311 $=$ SBS $1517+5017$ (Liebert et al. 1994), are spectrum binaries in which a DA spectrum is seen in the blue while that of a carbon star is evident in the red, thus providing strong support to the mass-exchange hypothesis. These two have small proper motions, $<0 . " 035 / \mathrm{yr}$, whereas most dCs have been identified by motions $>0 . " 1 / \mathrm{yr}$ which place an upper limit on their luminosities several magnitudes below the AGB.

De Kool \& Green (1995) and Green (2000) have estimated that the dCs may outnumber the AGB C stars per unit volume with $\sim 110$ halo dCs detectable to $V \sim 18$; however, only about $10 \%$ of this number are known and none has been found in the last few years. Since those known have been found without the benefit of a systematic search, it is difficult to draw firm conclusions when discussing their origin, frequency, and kinematics. One way to attempt to close this gap is to measure known $\mathrm{C}$ stars for appreciable proper motion. The relation between a star's absolute and apparent magnitudes, its tangential velocity and proper motion is

$$
M=m-5\left(\log V_{t}-1.68-\log \mu\right) .
$$

Thus, for a given apparent magnitude and measured proper motion, an assumed maximum tangential velocity leads to a limit on the star's luminosity if the star is to be bound to the Galaxy. For example, if a 15th magnitude $C$ star were found to have a proper motion of $0 . " 1 / \mathrm{yr}$, it could be no brighter than $M_{V} \approx+4.9$ unless it were escaping from the Galaxy. Such a star would be a dC.

\section{The Proper Motion Search}

We have begun a systematic program to search for motions among a large number of catalogued $\mathrm{C}$ stars subject to certain conditions. We use the archive of digitized scans made from the 1950-era POSS red (E) plates and the 1980-era V and SERC-J plates used for the HST Guide Star Catalogue which are available on $10 \times$-compressed CD-ROMs accessible on a jukebox at the Space Telescope Science Institute. The early-epoch scans are not available on CD south of $\delta-10^{\circ}$, so all of the stars examined for proper motion are north of that. In the magnitude range $12<V<18$, proper 
motions can be determined down to 35 milliarcseconds/yr (mas/yr) from these scans with a formal error of about \pm 10 mas/yr over the typical 30 year epoch difference. A more complete description of the procedures and software is given in MacConnell et al. (1995).

At the outset, we confined our program to stars in Stephenson's (1989) A General Catalog of Cool Galactic Carbon Stars, Second Edition which are at $|b| \geq 7^{\circ}$, not of types $\mathrm{R}$ or $\mathrm{CH}$, and within the declination and brightness limits specified above. We extracted $7 ! 3 \times 7$ ! 3 frames for both epochs on the resulting $\sim 300$ stars and ran Roberts' nearest-neighbor matching algorithm and proper-motion program; the average number of reference stars per field was about two dozen. We measured a further 30 high-latitude C stars resulting from the First Byurakan Survey and published by Abramyan and Gigoyan in Astrofizika in the years 1989-1995.

From analysis of this material, we thought we had found one new dC. No. 3635 in Stephenson's catalogue has a proper motion of $0 .{ }^{\prime \prime} 06 / \mathrm{yr}$; if its tangential velocity were of order $100 \mathrm{~km} \mathrm{~s}^{-1}$, its $M_{V}$ would be $\sim 5.3$. At $V \sim 13$, it would have been the brightest member of the dC class. However, at P. Green's request, J. Huchra obtained a spectrum of CCS 2-3635 with the FAST spectrograph at the 1.5-m telescope on Mt. Hopkins. The spectrum shows no characteristics of a $\mathrm{C}$ star but is a good match with M0 V. W. P. Bidelman kindly examined the Burrell Schmidt objective-prism plate on which the star was found and agrees that it should not have been published as a $\mathrm{C}$ star. We have continued with extractions on northern $\mathrm{C}$ stars within $7^{\circ}$ of the galactic plane. We report that among $1400 \mathrm{C}$ stars in the Stephenson catalogue meeting our criteria and of R.A. $<21^{h}$, as well as among the Byurakan stars, there are no new dCs, i.e. no stars having $\mu>0 . " 035 / \mathrm{yr}$.

\section{Dwarf S Stars?}

There are sound reasons to believe that dwarf S stars in mass-transfer binaries should also exist, but none is known. Subject to the same constraints as above, we searched for motions among 322 stars in Stephenson's (1984) $A$ General Catalogue of Galactic S Stars, Second Edition and additional ones published by him in 1990 and found one with sizeable motion: no. 1237 has $\mu=0$." $16 / y$ r, which is about $10 \%$ below the limit for Luyten's survey for the general field. W.P. Bidelman has also examined the spectrum of this star on the discovery plate at our request and finds that it does not have $\mathrm{ZrO}$ absorption at $6474 \AA$ but instead has strong $\mathrm{CaH}$ at $6385 \AA$; the two bands are separated by only about one resolution unit at the dispersion of the plate. Thus, no. 1237 joins the other ex-S stars, nos. 25, 780, and 875, which Stephenson (1986) found to be early M dwarfs with strong $\mathrm{CaH}$, 
making them possibly similar to Kapteyn's star (see Wing et al. 1976).

\section{References}

Dahn, C. C., Liebert, J., Kron, R. G., Spinrad, H. \& Hintzen, P. M. 1977, ApJ, 216, 757

Dearborn, D. S. P., Liebert, J., Aaronson, M., Dahn, C. C., Harrington, R., Mould, J. \& Greenstein, J. L. 1986, ApJ, 300, 314

de Kool, M. \& Green, P. J. 1995, ApJ, 449, 236

Green, P. J. 2000, in IAU Symp. 177: The Carbon Star Phenomenon, ed. R.F. Wing (Kluwer), p. 27

Green, P. J., Margon, B., Anderson, S. F. \& MacConnell, D. J. 1992, ApJ, 400, 659

Green, P. J., Margon, B. \& MacConnell, D. J. 1991, ApJ, 380, L31

Heber, U., Bade, N., Jordan, S. \& Voges, W. 1993, A \& $A$, 267, L31

Liebert, J., Schmidt, G. D., Lesser, M., Stepanian, J. A., Lipovetsky, V. A., Chaffee, F. H., Foltz, C. B. \& Bergeron, P. 1994, ApJ, 421, 733

MacConnell, D. J., Roberts, W. J. \& Williamson, R. L. II 1995, in IAU Colloquium 148: The Future Utilisation of Schmidt Telescopes, ed. J. Chapman, R. Cannon, S. Harrison and B. Hidayat, ASP Conf. Ser., 84, 224

Stephenson, C. B. 1984, Publ. Warner \& Swasey Obs., 3, 1

Stephenson, C. B. 1986, PASP, 98, 467

Stephenson, C. B. 1989, Publ. Warner \& Swasey Obs., 3, 53

Wing, R. F., Dean, C. A. \& MacConnell, D. J. 1976, ApJ, 205, 186 